\title{
Review of gold reef sampling and its impact on the mine call factor
}

\author{
by A. Fourie* and R.C.A. Minnitt ${ }^{\dagger}$
}

\section{Synopsis}

Sampling error and bias, especially the Increment Preparation Error (IPE), are introduced when the tool allocated to do the job fails to extract a representative sample. This is the case with chip sampling; the tool can only extract haphazard shapes of loose or fractured material and not always from within the demarcated sample area. Increment Extraction Error (IEE) and Increment Delimitation Error (IDE) could be severe, but are found to be relatively unbiased. The uncontrolled action of the sampler in discarding excess sample material after sample collection has a significant impact on the error and bias introduced with each and every extraction. A new type of bias, referred to as the 'waste discard bias', arises when samplers select what portion of the excess sample material to discard at the sample site. Material visually identified as waste is discarded in preference to mineralized broken ore if there is an excess of sample material. This results in a considerable error in the analytical results. The bias is proportional to the reef-waste ratio in the demarcated sample.

Keywords

sampling, error, bias, mine call factor.

\section{Introduction}

Two types of 'sampling' are distinguished, namely sampling of particulate materials (broken ores) and sampling of solid, unbroken in situ mineralized reef. This study considers only chip- or face-sampling of in situ, goldbearing, carbon-rich conglomerates typical of the Upper Witwatersrand Supergroup and examines where components of sampling error and bias, clearly specified in the Theory of Sampling, may arise during the practice of 'face sampling'. Many millions of face samples have been extracted from Witwatersrand-type mines using a hammer and chisel, and detailed descriptions (standard operating procedures) of how the samples should be taken have been developed by many mining companies. There is, however, no taxonomy or nomenclature for managing or reporting the different types of errors associated with chip sampling. The Theory of Sampling compiled by Pierre Gy in the early 1950 s provides, comprehensive nomenclature of sampling errors that is applied to particulate sampling of ore in a lot, a stockpile, or a moving stream on a conveyor belt. The taxonomy and characteristics of sampling errors, grouped according to the sources from which they arise, and identified by Gy $(1973,1979,1982,1992,1995$, and 1998) are shown in Figure 1. This classification of errors is used extensively in regard to the sampling of particulate materials in the mining and minerals industry, but not in regard to chip sampling. A relatively small mass (hundreds of grams) of representative particulate material for estimating the average grade of a much larger mass (thousands of tons - the 'lot' in the Theory of Sampling) is extracted by the chipping or cutting of a representative rock 'sample' from the stope face.

While differences between the sampling of particulate materials and chip sampling from solid, in situ reef are emphasized, there are aspects of the Theory of Sampling that can also be applied to face sampling. For example, face sampling is unaffected by the Fundamental Sampling Error (FSE) or the Grouping and Segregation Error (GSE), making a consideration of these errors and bias related to material characterization (Errors 2 and 3, Figure 1) irrelevant in the sampling process. In addition, the errors associate with interpolation and periodicity (Errors 4 and 5, Figure 1) are not relevant in face sampling. In the Theory of Sampling, bias due to errors in delimitation, extraction, preparation, and weighting (referred to as an error in most literature) of the samples arises due to the interactions between the particulate materials and the sampling equipment. This is also true for the chipping of samples from solid mineralized rock, and is therefore applicable to face sampling.

* HOD Evaluation, Target Gold Mine, Welkom, South Africa.

+ School of Mining Engineering, University of the Witwatersrand, Johannesburg, South Africa.

(c) The Southern African Institute of Mining and Metallurgy, 2016. ISSN 2225-6253. Paper received Mar. 2016; revised paper received Jul. 2016. 


\section{Review of gold reef sampling and its impact on the mine call factor}

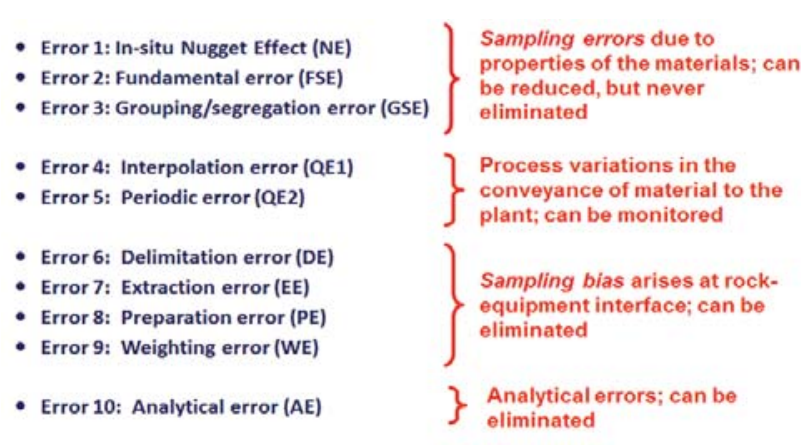

Figure 1-The ten sampling errors of Pierre Gy

\section{Errors and bias associated with chip sampling}

This study investigates the error and bias that manifest as a consequence of poor sample extraction, by simulating and testing the conditions in which actual underground sampling takes place. In addition, the intuitive and responsive behaviour among samplers that is thought to be responsible for introducing bias during and after sample collection is considered.

Early investigations into underground sampling procedures on carboniferous reefs identified what Fourie (2015) termed a 'soft-reef' bias. The contribution of soft-reef bias to the generation of significant sampling error and bias is the result of over-extraction of soft, carbon-rich material compared to the harder adjacent host quartzite and conglomerate within the demarcated sample area (see Figure 2). This form of bias always results in an overestimation the grade of the in situ sample. (Cawood, 2003; Lerm, 1994; Sichel, 1947).

In addition, the inclusion of waste in the demarcated sample area was also identified as a source of error and bias (Fourie, 2015). A review of work by early investigators of sampling issues on Witwatersrand mines (Cawood, 2003) indicated a preference for attributing overestimation of grade to sampling bias, rather than a comprehensive understanding of the source and causes of bias and error in general. Cawood concluded that what Fourie (2015) terms the 'softreef bias' may result in overestimation of the overall grade by as much as $26 \%$, due to over-extraction of softer carbon-rich material and poor estimation of the actual reef width. An example of how the bias is introduced is graphically illustrated in Table I.

Historically, the perception that chip sampling is responsible for overestimation of the in situ grade is an argument used by mining personnel as an easy and obvious explanation as to why some shafts reported low mine call factors (MCFs) ${ }^{1}$. The problem with attributing the low MCF to over-sampling and soft-reef bias is that the search for other contributors to the problem is neglected. As a result, the actual sources of error responsible for the deterioration of the MCF, such as dilution, sweepings, and incorrect tramming, remain undetected.

There is no dispute that oversampling of the softer portions of the reef introduces error and bias leading to low MCF, but such sampling errors would have to be introduced at every sampling site and during the extraction of every sample. The possibility of this happening in practice is unrealistic. The existence of the soft-reef bias was confirmed during underground investigations of sampling performance, but this type of bias was observed only once out of a total of 120 samples extracted. It is also likely that some of the in situ carbon exposed in the reef face will be lost as blast fragments impinge on the face. Further losses may occur when the face is washed down and during barring of loose rock from the face before sampling.

The thickness of the carbon seam in the stope face also contributes to the incidence of soft-reef bias, but it is very rarely reported in the records kept by samplers; even today it is not recorded. The chisel blade used to extract the sample is too wide to allow for extraction of thin carbon seams. Only if the carbon seam is $1 \mathrm{~cm}$ or more thick could the carbon be easily extracted. It is unlikely that the use of a chisel would result in over-extraction of carbon material from seams less than $3 \mathrm{~mm}$ thick. In areas where illegal mining takes place, the zama zamas use 8-gauge wire to selectively (gouge) burrow out the soft carbon.

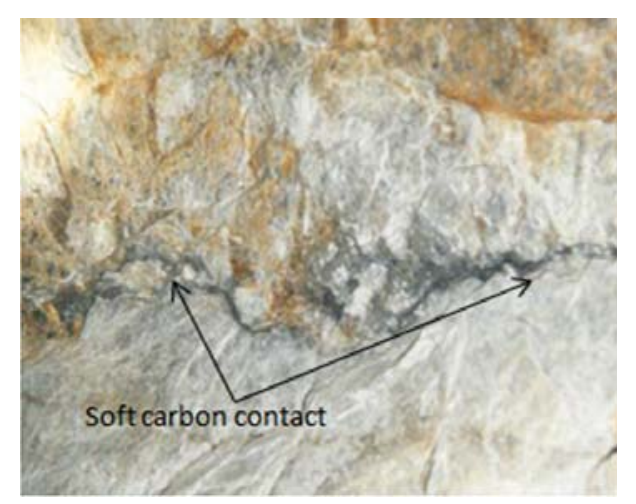

Figure 2-A narrow carbon-rich zone exposed just below the hangingwall (Fourie, 2015, p. 68)

\begin{tabular}{|c|c|c|c|c|}
\hline \multicolumn{5}{|c|}{$\begin{array}{l}\text { Table I } \\
\text { Oversampling of the contact affecting the grade } \\
\text { (Cawood, 2003, p. 24) }\end{array}$} \\
\hline Category & $\begin{array}{l}\text { ChW } \\
\text { (cm) }\end{array}$ & $\begin{array}{c}\text { Grade } \\
(\mathrm{g} / \mathrm{t})\end{array}$ & $\begin{array}{l}\text { Value } \\
\text { (cm.gt) }\end{array}$ & $\begin{array}{c}\text { Sample shape } \\
\text { (section) }\end{array}$ \\
\hline Waste above reef & 1 & 0 & 0 & \\
\hline Reef & 10 & 150 & 1500 & \\
\hline Waste below reef & 0 & 0 & 0 & \\
\hline Recorded channel & 11 & 136 & 1500 & \\
\hline
\end{tabular}




\section{Review of gold reef sampling and its impact on the mine call factor}

This observation suggests that the incidence of soft-reef bias in reefs carrying thin carbon seams is far less frequent than previously thought. Furthermore the frequency of this type of bias is not sufficient to cause the deterioration of the MCF. This observation was confirmed by AngloGold Ashant (Freeze et al., 2013) in research to investigate the soft-reef bias and the role of chip sampling as agents responsible for the declining MCF. Statistical comparisons between samples extracted by chip sampling compared to 'coffin sampling', thought to be the 'perfect sample', did not find any consistent bias. In some areas chip sampling indicated undersampling and in other areas oversampling, but within the variability of the accumulation value of the reef (Table II).

Textbook examples of perfect coffin samples were compared with chip sampling, for which the extraction was not so perfect. It was anticipated that because of the existence of the soft-reef bias the mean grade of the chip samples should be higher than that of the coffin samples. In Table II the average cm.g/t in column 'Cut Coffin Paired A' for the $430 E D$ area is lower than that of the 'Chip Paired' data. The 'Cut Coffin Paired B' sample for the same area is higher than the 'Chip Paired' samples. The 'Cut Coffin Paired C' mean is lower than the corresponding 'Chip Paired' data. Area 440ED shows a significantly higher grade for all 'Cut Coffin Paired' data compared to the corresponding 'Chip Paired' data. In the area 460W ED, 'Chip Paired' data is significantly higher than the corresponding 'Cut Coffin Paired' data. Flitton (2011) concluded that the comparisons did not indicate any bias, and the also confirmed that chip sampling actually compared well with alternative methods capable of extracting better samples.

It was argued that chip sampling carried out under careful supervision produced samples of better quality than could normally be expected. This implies that samplers under supervision extract better quality samples or are doing something other than they normally do during or after sample extraction. The fact that samplers have a free hand in adjusting both the sample mass and the sample content in the period between taking the sample and it reaching the surface is a completely new insight. The process of discarding sample material after extraction because there was an excess of sample material, was observed by the principal author during the underground investigations.

As a result of these studies AngloGold Ashanti in 2012 introduced the so-called Quality Index, a means by which the quality of the chip samples that were collected was improved. The company found that the average mass of the samples increased considerably 'after' the implementation of the Quality Index (2012-2013) compared to the 'before' situation (2011), as shown in Table III.

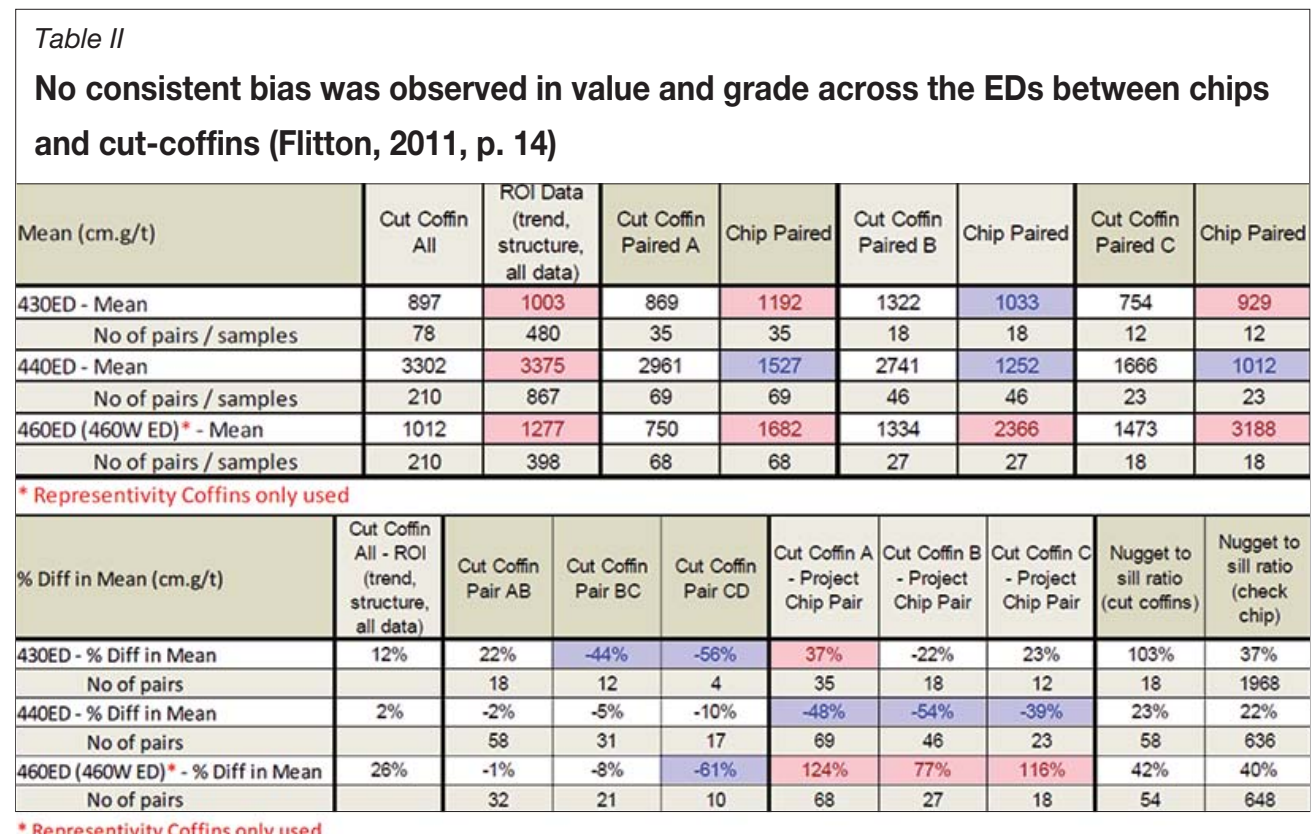

\footnotetext{
Table III
}

Minimum and maximum sample mass before (2011) and after (2012-2013) implementation of the 'Quality Index' (Fourie, 2015, p. 125)

\begin{tabular}{|c|c|c|c|c|c|c|c|}
\hline Year & $\begin{array}{c}\text { Average mass of } \\
\text { samples }\end{array}$ & $\%$ Difference & $\begin{array}{c}\text { Maximum mass of } \\
\text { samples }\end{array}$ & $\%$ Difference & $\begin{array}{c}\text { Minimum mass of } \\
\text { samples }\end{array}$ & $\%$ Difference & $\begin{array}{c}\text { No } \\
\text { samples }\end{array}$ \\
\hline $\begin{array}{l}2011 \\
2012 \\
2013\end{array}$ & $\begin{array}{l}360 \\
595 \\
611\end{array}$ & $\begin{array}{c}0 \% \\
39 \% \\
3 \%\end{array}$ & $\begin{array}{c}591 \\
1137 \\
1112\end{array}$ & $\begin{array}{c}0 \% \\
48 \% \\
-2 \%\end{array}$ & $\begin{array}{l}226 \\
291 \\
294\end{array}$ & $\begin{array}{c}0 \% \\
22 \% \\
1 \%\end{array}$ & $\begin{array}{c}127 \\
775 \\
1001\end{array}$ \\
\hline
\end{tabular}




\section{Review of gold reef sampling and its impact on the mine call factor}

The change in sample mass before and after the introduction of the Quality Index is shown graphically in Figure 3. According to AngloGold Ashanti the increase in sample mass is considered to be due to improved sample extraction as a result of stricter control on sampling either by a supervisor or by comparing photographs of the sample site taken before and after sample extraction. However, the time taken to extract a sample remained the same irrespective of whether it was taken before or after the implementation of the Quality Index. The question we now need to consider is, did the sample mass increase due to better extraction or simply because samplers were no longer at liberty to discarded material at will? If extraction had been improved, a significant increase in the time taken to extract a sample would have been expected because the method and the tools for extracting the sample remained unchanged. (Freeze et al., 2013).

According to the Quality Index, the evaluator is required to rate sample extraction as acceptable or unacceptable by comparing photographs taken before and after sampling. From an inspection of the 'before' and 'after' photographs shown in Figure 4 it is difficult to tell firstly, how the observable differences were used to make an 'acceptable' or 'unacceptable' decision, and secondly what the reasons for the increase in the average sample mass (Figure 3) were. In addition, the time difference between the 'before' (09:42) and 'after' (09:44) photographs (Figure 4a and 4b) indicates that only two minutes was spent extracting the sample, suggesting that the quality of the sampling procedure did not meaningfully improve. We suggest that a possible reason for the observed increase in the average sample mass after implementation of the Quality Index (as indicated in Figure 3 ) is that none of the sample material was discarded after it was collected.

Careful reading of the standard operating procedures indicates that the sampler's habit of discarding excess sample material in the stope immediately after taking the sample was the only activity not controlled by the standard operating procedures. In order to comply with the rules of the newly introduced Quality Index, samplers were therefore not discarding excess material. In addition, data from Tshepong mine indicated that the average mass of the chip sample extracted was inversely proportional to the number of samples collected per sampler per shift. This led to the

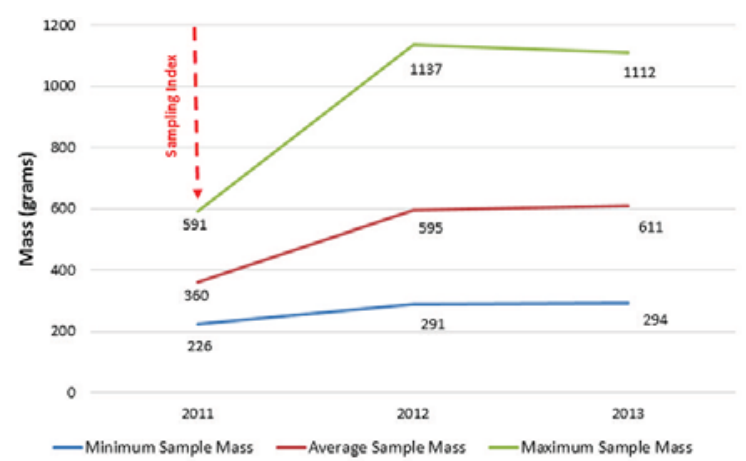

Figure 3-Change in the average mass of chip samples before and after implementation of the 'Quality Index' (Fourie, 2015, p. 125) intuitive, but important conclusion that the average sample mass decreased as the number of samples extracted per sampler per shift increased.

Prior to the introduction of the Quality Index, the amount of material sent to the assay laboratory depended not only on the amount material extracted, but also on the amount discarded after sample extraction. Since discarding of material, and as a result preferential selection of the betterlooking pieces of reef, occurred at every sample site this action became the prime suspect as a source of both error and bias. This is a possible reason why no bias was discernible between the chip samples and the 'coffin samples' analysed by Flitton and Freese (2013).

\section{Identification of the 'waste-discard' bias}

The 'waste-discard' bias has inadvertently been responsible, through human agency, for a bias towards higher grades for many years. If the samplers were unbiased in their selection of the excess sample material to be discarded this type of bias would not have arisen. Once the nature of this bias was identified, a five-question survey was conducted amongst 76 participants in order to determine if a bias existed because samplers were selective in discarding what they considered to be excess sample material. The questions are listed in Table IV and were designed to be simple, but also so that definitive answers in regard to the sampling procedures could be obtained. A compilation of the survey results is shown in Table V and Figure 6.

The answers obtained from samplers in regard to the procedures they tend to follow are listed in Table VI.

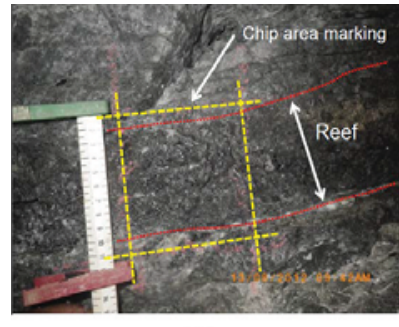

(a)

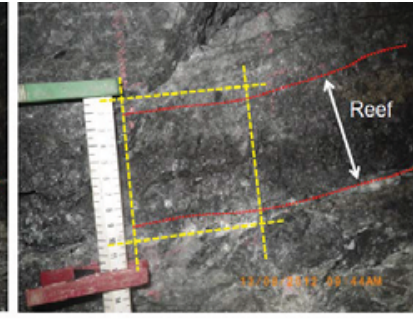

(b)
Figure 4-Photographs of the sample face used to rate the quality of a sample from the condition of the face (a) before and (b) after chipping (Freeze et al., 2013)

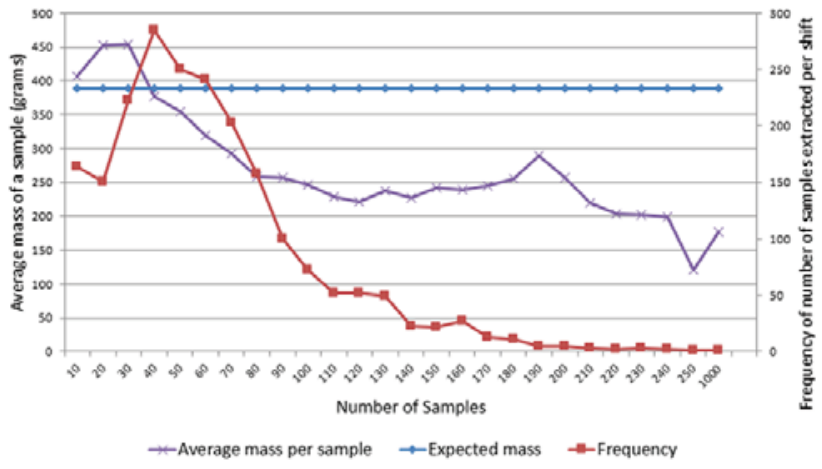

Figure 5-The sample mass is inversely proportional to the number of samples taken per shift (Fourie, 2015, p. 128) 


\section{Review of gold reef sampling and its impact on the mine call factor}

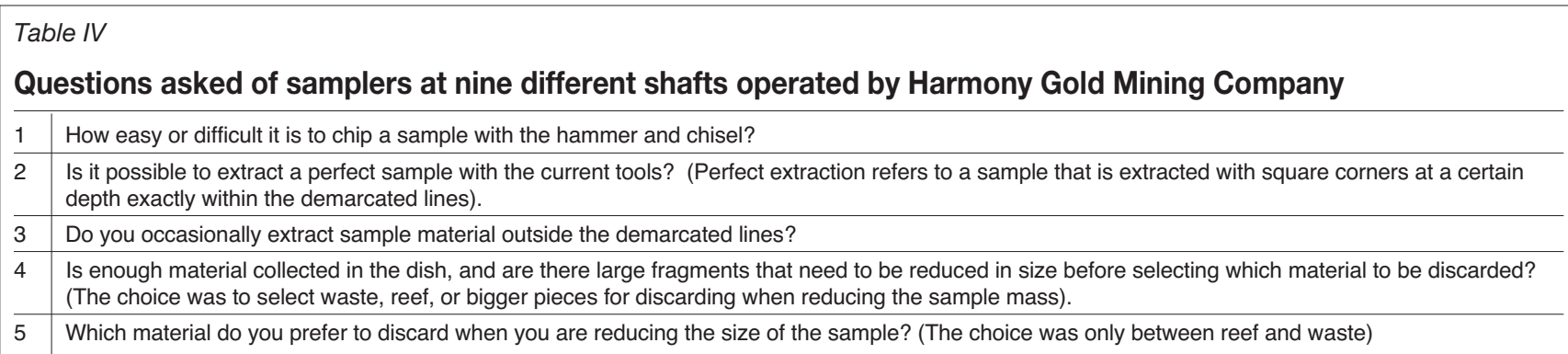

Table $\mathrm{V}$

Compilation of the results of the survey of samplers' answers of the five questions listed in Table IV

\begin{tabular}{|c|c|c|c|c|c|c|c|c|c|c|c|c|c|c|}
\hline \multirow[b]{2}{*}{ Shaft } & \multicolumn{3}{|c|}{ Question 1} & \multicolumn{3}{|c|}{ Question 2} & \multicolumn{3}{|c|}{ Question 3} & \multicolumn{3}{|c|}{ Question 4} & \multicolumn{2}{|c|}{ Question 5} \\
\hline & Easy & Difficult & $\begin{array}{c}\text { Very } \\
\text { difficult }\end{array}$ & Yes & No & Sometimes & $\begin{array}{l}\text { Most of } \\
\text { the times }\end{array}$ & Sometimes & Never & Reef & Waste & $\begin{array}{l}\text { Biggest } \\
\text { pieces }\end{array}$ & Reef & Waste \\
\hline Target1 & $17 \%$ & $67 \%$ & $17 \%$ & $0 \%$ & $100 \%$ & $0 \%$ & $17 \%$ & $83 \%$ & $0 \%$ & $33 \%$ & $33 \%$ & $33 \%$ & $83 \%$ & $17 \%$ \\
\hline Target 3 & $0 \%$ & $33 \%$ & $67 \%$ & $33 \%$ & $0 \%$ & $67 \%$ & $33 \%$ & $0 \%$ & $67 \%$ & $0 \%$ & $100 \%$ & $0 \%$ & $100 \%$ & $0 \%$ \\
\hline Tshepong & $29 \%$ & $57 \%$ & $14 \%$ & $29 \%$ & $43 \%$ & $29 \%$ & $14 \%$ & $57 \%$ & $29 \%$ & $29 \%$ & $57 \%$ & $14 \%$ & $100 \%$ & $0 \%$ \\
\hline Phakisa & $40 \%$ & $50 \%$ & $10 \%$ & $20 \%$ & $50 \%$ & $30 \%$ & $0 \%$ & $20 \%$ & $80 \%$ & $0 \%$ & $80 \%$ & $20 \%$ & $100 \%$ & $0 \%$ \\
\hline Bambanani & $50 \%$ & $50 \%$ & $0 \%$ & $17 \%$ & $33 \%$ & $50 \%$ & $17 \%$ & $50 \%$ & $33 \%$ & $0 \%$ & $67 \%$ & $33 \%$ & $100 \%$ & $0 \%$ \\
\hline Joël & $0 \%$ & $92 \%$ & $8 \%$ & $17 \%$ & $33 \%$ & $50 \%$ & $0 \%$ & $67 \%$ & $33 \%$ & $8 \%$ & $75 \%$ & $17 \%$ & $92 \%$ & $8 \%$ \\
\hline Unisel & $8 \%$ & $58 \%$ & $33 \%$ & $0 \%$ & $67 \%$ & $33 \%$ & $25 \%$ & $67 \%$ & $8 \%$ & $0 \%$ & $83 \%$ & $17 \%$ & $92 \%$ & $8 \%$ \\
\hline Masimong & $17 \%$ & $58 \%$ & $25 \%$ & $17 \%$ & $50 \%$ & $33 \%$ & $8 \%$ & $75 \%$ & $17 \%$ & $0 \%$ & $67 \%$ & $33 \%$ & $83 \%$ & $17 \%$ \\
\hline Kusasahlethu & $13 \%$ & $63 \%$ & $25 \%$ & $13 \%$ & $50 \%$ & $38 \%$ & $0 \%$ & $88 \%$ & $13 \%$ & $0 \%$ & $100 \%$ & $0 \%$ & $88 \%$ & $0 \%$ \\
\hline Totals & $19 \%$ & $59 \%$ & $22 \%$ & $16 \%$ & $47 \%$ & $37 \%$ & $13 \%$ & $56 \%$ & $31 \%$ & $8 \%$ & $74 \%$ & $19 \%$ & $93 \%$ & $6 \%$ \\
\hline
\end{tabular}

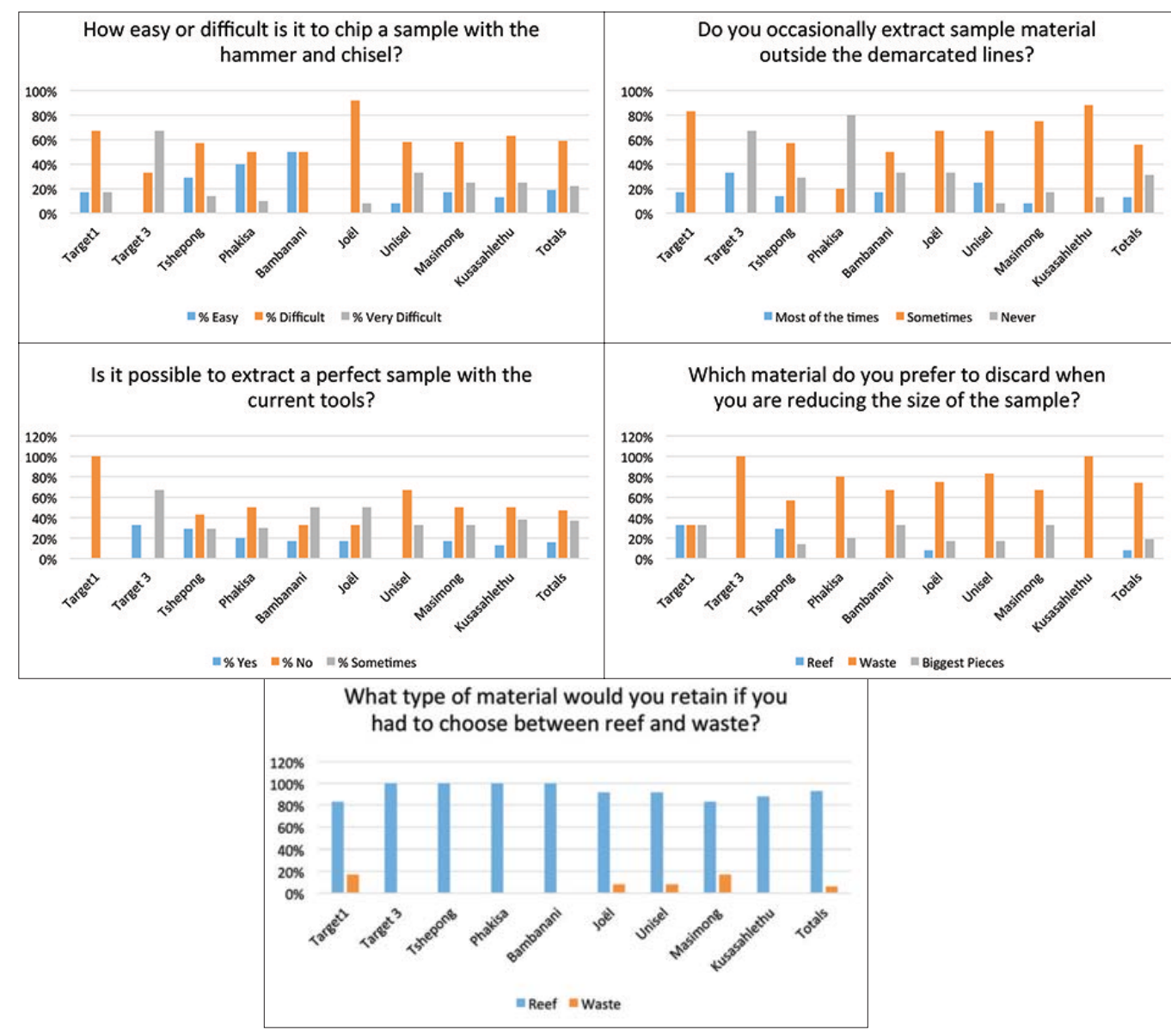

Figure 6-Graphical representation of the survey results 


\title{
Review of gold reef sampling and its impact on the mine call factor
}

\author{
Table VI \\ A compilation of the answers from the participants in the questionnaire survey

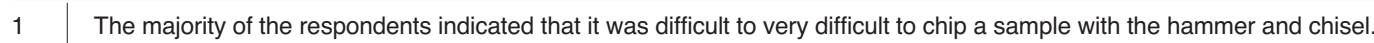 \\ $2 \quad$ Almost $50 \%$ of the respondents did not think that perfect extraction of a sample with the current tools is possible. \\ $3 \quad$ The majority of participants answered 'No' or 'Sometimes' when asked about occasionally extracting sample material outside the demarcated lines. \\ These answers provide a good indicator of the degree of difficulty involved extracting a representative sample. \\ $4 \quad$ The majority of participants answered 'No' or 'Sometimes' when asked if they managed to collect enough material in the dish and if there were large \\ fragments that needed to be reduced in size before selecting which material to be discarded. Answers to this question also provided a good indication \\ of the degree of difficulty involved extracting a representative sample. \\ $5 \quad$ Surprisingly, almost $93 \%$ of samplers said that they would keep the reef and discard the waste when asked which type of material they would retain if \\ they had to choose only between reef and waste. Although this question is very similar to Question 4, it isolated and emphasized the inherent bias \\ amongst samplers towards keeping the reef.
}

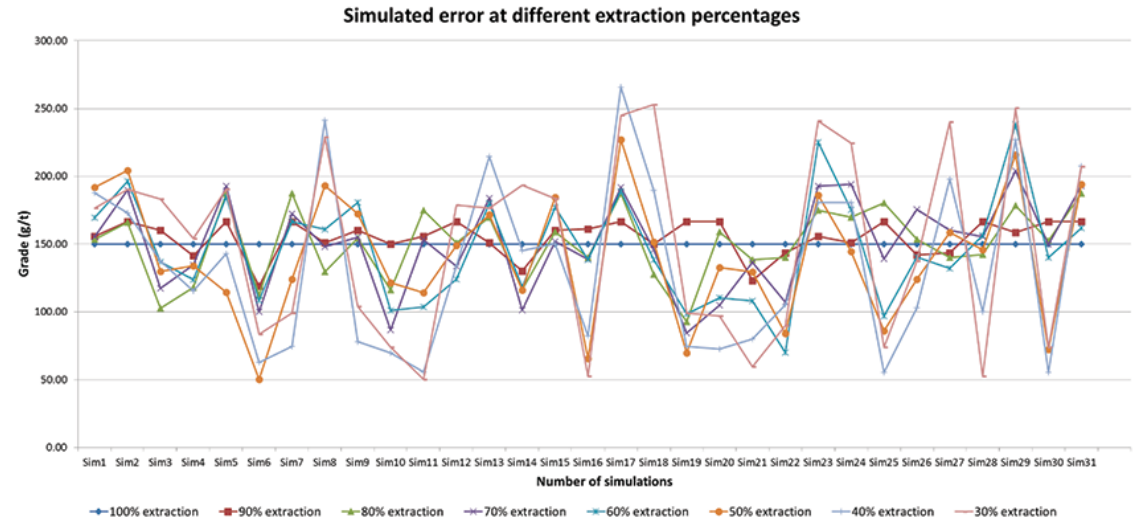

Figure 7-Graphical illustration of how the percentage material extracted from a delimited sample affects the precision of the grade (source: Fourie, 2015 p. 106)

The results of the survey shown in Tables V and VI indicate that samplers not only found sample extraction with the hammer and chisel difficult (question 1), but $93 \%$ of the respondents indicated that they select waste rather than reef when faced with the choice of what to discard (question 5).

The results for a simulated sample extraction model designed by Fourie (2015) are shown in Figure 7, and the average values are also represented by the red curve. The data indicates that even though the levels of extraction vary from $30 \%$ to $100 \%$, and estimates of gold grade vary positively and negatively around the average of $150 \mathrm{~g} / \mathrm{t}$, they did not introduce any bias.

The graphs for different levels of extraction (30\% to $100 \%$ in Figure 7 ) indicate that highly heterogeneous sample compositions were on average unbiased, irrespective of the quality of extraction. The same simulation was also used to test the effect of selective waste discard after extraction. In the case of the simulations the bias was kept constant at around $18 \%$ of waste being discarded. In this case the bias is constant and directly proportional to the waste. However, it is clear from actual data collected from Tshepong mine that the percentage of material being discarded varies and is proportional to the number of samples collected per day as shown in Figure 5.

The results of the questionnaire survey (Table VI) indicate that preferential discarding of waste portions from a broken ore sample results in a significant bias responsible for overestimating the grade of the extracted material. Even the most perfectly extracted sample will overestimate the true in situ grade if this error is introduced by preferentially discarding waste. In Table VI the total percentage error after 31 simulations was $-1.42 \%$, indicating a slight negative bias. The same extraction error mass was discounted by $18 \%$ waste to simulate selective sample reduction after collection. The total percentage error now changed from slightly negative to a positive $20.22 \%$ (Table VII).

Table VII is split into two portions; the first deals with the extraction error simulated at different percentages of extraction, and the second deals with the same simulations except with an $18 \%$ reduction in the amount of waste material. The figures in the first portion are the results of the original simulations done at different percentages of extraction of unbiased portions of a sample (column on the left), while the right-hand side of the table shows the same results, except that $18 \%$ waste was deducted from each simulated example. As a result, the line with 100\% extraction also shows a bias even though it was extracted perfectly. These results are depicted graphically in Figure 8.

\section{Discussion}

This 'waste-discard' bias severely affects reefs with a high waste-to-reef ratio such as the carboniferous Basal Reef and the Carbon Leader Reef. Generally the carboniferous reef is relatively thin, and hence most of the channel samples can easily cover the entire reef in a single sample. In such cases 


\section{Review of gold reef sampling and its impact on the mine call factor}

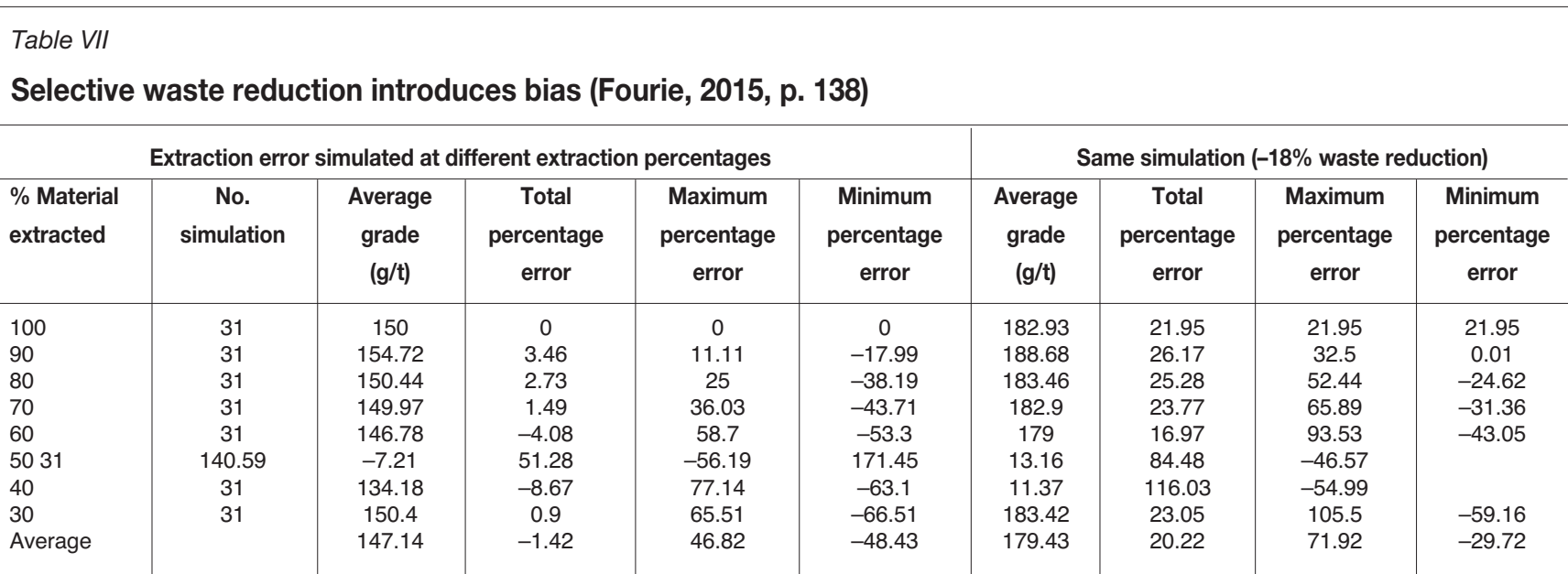

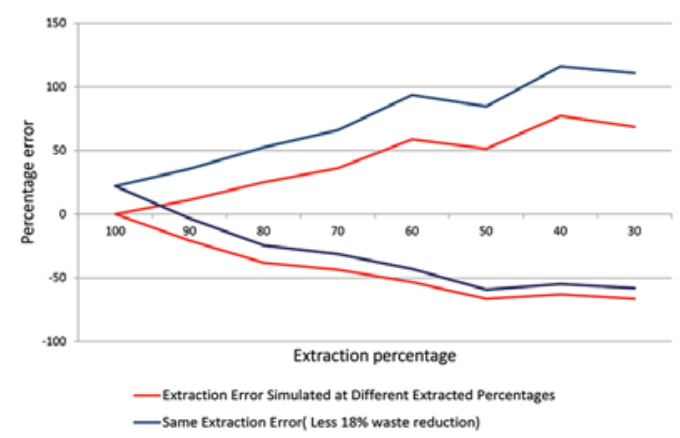

Figure 8-Selective discarding of waste responsible for bias (Fourie, 2015, p. 138)

the high waste-to-reef ratio tends to amplify the wastediscard bias, which now becomes severe. In fact, the bias generated from this error now resembles the soft-reef bias, but unlike soft-reef bias - which is introduced during extraction - the waste-discard bias is due to a post-extraction material selection procedure that introduces a bias.

It is evident from the simulated model that as the composition of the sampled reef becomes more homogenous, the effect of 'selective discard' diminishes. By changing the reef-to-waste ratio in the simulation models of possible overand under-extraction, it was found that the percentage extraction error changed accordingly. Further experimentation revealed that the percentage error in the gold grade tends to decrease as the sample composition becomes more homogenous, provided the extraction error remains constant. 'Extraction error 1', simulated in Figure 9, explains the rationale behind this simulation. 'Extraction error 1' had a reef-to-waste ratio of $14.29 \%$ based on a $1 \mathrm{~cm}$ reef and $6 \mathrm{~cm}$ waste portion and incomplete extraction, resulting in an a grade error of $40 \%$ (Table VII).

The reef thickness was incrementally increased and the waste proportionally decreased, as shown in Figure 9. The percentage extraction error was kept the same, the simulated chip sample model was updated with changes in the component parts of reef and waste, and a new expected grade was calculated for each step change. The methodology was applied in order to calculate the changes in gold grade error as a result of changes in the homogeneity of the reef components from zero to $100 \%$, while maintaining exactly the same amount of extraction error (Figure 9). Figure 9 illustrates the idea that the amount of error decreases as the homogeneity of the material being sampled increases; this usually happens with chip sampling.

If the exposed stope faces consist mainly of conglomerates with little internal waste, then the sample is demarcated according to observed lithology, i.e. reef and waste. In such a case the extracted material will consist of either reef or waste. When sampling the conglomerates the sampler and his team will select only bigger pieces of reef to discard since there will be no waste present; therefore it is unlikely that the discards will introduce a bias. The selection process in such a case will be totally random and free from bias. When extracting a sample demarcated in the waste portion, the tendency of the samplers is to discard any reef (rather than waste) and/or the bigger pieces of waste. This may explain why shafts that mine lithologically homogenous reefs experience a better MCF than those mining carboniferous reefs, despite the extraction errors being fairly similar.

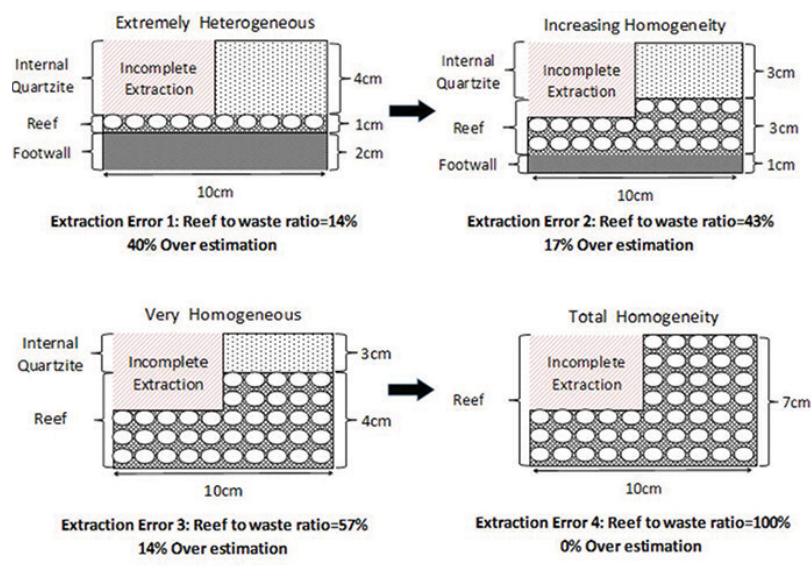

Figure 9-Percentage extraction error decreases as the sample becomes more homogenous (Fourie, 2015) 


\section{Review of gold reef sampling and its impact on the mine call factor}

This 'waste-discard bias' error may also be the source of higher grade in samples that include a larger proportion of waste, rather than a decrease as might be expected because of dilution. This is because extra waste from a correctly demarcated and extracted sample will be selectively discarded. This results in a grade similar to a sample extracted with no waste included, giving the perception that the error was as a result of inclusion of waste.

The percentage error introduced with the "waste-discard bias' error depends on the reef-to-waste ratio of the demarcated sample, and on the percentage of waste discarded after extraction. It is impossible to determine what the percentage of discarded waste is, because extraction is never constant and discarding is randomly determined by the sampler and the number of samples collected during the shift. The authors believe strongly that this error, as well as other errors such as 'soft-reef' bias, are major contributors to the bias that results in overestimating the grade. The only way to prevent this error completely would be to develop a tool that is able to extract a sample exactly to the shape and volume demarcated on the face. Since no such tool exists, the only way to mitigate this bias will be to discourage any form of material discarding after the initial sample extraction. However, this is easier said than done in practice because occasionally samples may include material outside the demarcated area that needs to be discarded. AngloGold Ashanti has shown that improved focus on standards, one of which included an increase in sample mass, resulted in samplers being discouraged from discarding any material after extraction. Since it is assumed that the increase in mass was not necessarily as a result of improved extraction, it is safe to conclude that the bias expected with 'selective discard' was kept to a minimum, leading to an improvement in the MCF.

There is evidence in the literature (Beringer, 1938; Cawood, 2003; de Jager, 1997; Harrison, 1952; Hallbauer and Joughin, 1972; Lerm, 1994; Magri and Mckenna, 1986; Muller, 1950; Sichel, 1961; Storrar, 1987) that our predecessors acknowledged the existence and introduction of bias during chip sampling, especially on the thinner carboniferous reefs. Methods introduced to minimize the error included selective capping and even the exclusion of results during the estimation process, which helped to lower the 'gold called for' and improving the MCF. This allowed the responsible persons to reduce the $\mathrm{MCF}$, but it failed to solve the problem; it was purely a cosmetic exercise. The authors are not suggesting that MRMs revert to the earlier outdated practices of sample exclusion or capping. This mitigation of the effect of extreme outliers is accommodated in the kriging algorithms, but soft-reef and waste-discard bias can never be eliminated; they are forever embedded in a bias of the sample data. Only when the bias is fully understood and mitigated will it be possible to identify other factors that may actually be responsible for the deterioration of the MFC. Current estimation methods make provision for capping of data during variography and estimation, but this only eliminates the extreme errors. The question is: 'do the automatic biasadjusting algorithms in the kriging equations adjust sufficiently for the bias present in the bulk of the sample data?' It should be accepted that the data on some mines is more affected by error and bias. We should acknowledge that our current estimation methods may not successfully ameliorate the problem of bias inherent in the data. Even if the new sampling tools and equipment could minimize the bias, the sampling error and bias inherent in the historical data will still influence the estimation.

It is therefore important that implementation of sampling improvement initiatives such as the 'Quality Index' should be continued and adjusted if early results do not indicate an improvement in the estimation of resources and reserves. The impact on the MCF may take time to manifest, but it is likely that it will improve in the longer run and that the amount of improvement will be equivalent to the bias introduced by sampling that was present before implementation. The focus can then be shifted to other areas, such as the assay methods, to improve the MCF even further. Unfortunately, it is not uncommon for more than one initiative to be introduced simultaneously to improve the MCF for a certain shaft, especially if the shaft is under investigation. If the MCF does improve, it is often difficult to determine which of the many interventions contributed the most to improvement.

Chip sampling, owing to its in situ extraction deficiencies, is probably one of the worst sample recovery methods, but in the difficult, confined, hot and unwelcoming environment in which samplers are expected to operate, it is probably the only consistently reliable and cost-effective method for underground face sampling. Despite its poor extraction capabilities, if no bias is introduced, it is the best available method. A comparative study with other methods has shown that, under certain conditions, chip sampling is still considered the best sampling method.

\section{Conclusion}

This research has demonstrated that the true source of error and bias introduced with a particular sampling method is misunderstood and misidentified. This arises because behavioural factors among the samplers, specifically the opportunity to selectively discard material from the broken ore, leads to the introduction of errors that are not properly identified and understood. These factors may change considerably from shaft to shaft, reef to reef, and from one sampling method to another. 'Soft-reef bias' has always been considered to be the main contributor of error when sampling the thin carboniferous reef types. It is believed to be responsible for overestimation of the grade, and this led to the conclusion that the only way to eliminate soft-reef bias was to develop a tool that will ensure perfect extraction. After many years of concerted effort and vast amounts of expenditure, no workable solutions have been identified. It is now evident that unless the person(s) responsible for sampling understand the true source of sampling errors, especially the bias that is associated with each and every sampling method, little improvement can be expected. If this awareness is not created then the direct result will be greater uncertainty in the assay results, which will inevitably lead to significantly unrealistic expectations, increased costs, and time-consuming frustration.

This investigation identified the 'waste-discard error' as the most likely reason for the introduction of bias and of overestimation when sampling thin carboniferous reefs. A 


\section{Review of gold reef sampling and its impact on the mine call factor}

survey among samplers confirmed that selective discard of waste after extraction introduced this error. Since it is not a result of poor extraction it can be immediately minimized with relatively little effort and cost through proper training, education, and strict adherence to sampling protocols. A further lesson learned is that any attempt to decrease costs by changing the sampling protocols, especially the sample mass reduction methodology, should be carefully considered. Changes to sampling protocols that are not carefully considered may introduce error and bias further down the sampling procedural chain that are more costly and detrimental than the saving envisioned (Minnitt, 2007). We should also be aware of Goethe's caveat: 'It is easier to perceive error than to find truth, for the former lies on the surface and is easily seen, while the latter lies in the depth, where few are willing to search for it.' (Zubko, 2003,p. 487).

\section{References}

Beringer, B. 1938. Underground Practice in Mining. 2nd edn. Mining Publications Ltd., London.

CAwooD, F.T. 2003. Underground face sampling on narrow gold reefs. Sampling practice and its impact on the mine call factor on Witwatersrand gold mines: lessons learned. Journal of the Institute of Mine Surveyors of South Africa, vol. XXXI, no. 7. pp. 202-216.

DE JAGER, E.J. 1997. The analysis of the mine call factor in gold mining, with specific reference to Western Holdings Mine. PhD thesis, University of the Witwatersrand, Johannesburg.

Freeze, B., Flitton, T., and Pillay, A. 2013 quality control and assurance of underground chip sampling at Kopanang Mine, South Africa. Proceedings of Sampling and Analysis: Best-practice in African Mining 2013, Misty Hills, Muldersdrift. Southern African Institute of Mining and Metallurgy, Johannesburg.

FLitTon, T.M. 2011. Review of chip and coffin samples taken at Kopanang Mine. Geoscience Technical Office (GTO) REP2011-1696. 14 pp.

FouRIE, D. and MinNiTT, R.C.A. 2013. The simulated chip-sample model as a method for quantifying error and bias in sampling thin carboniferous reef type, South Africa. Proceedings of Sampling and Analysis: Best-practice in African Mining 2013, Misty Hills, Muldersdrift. Southern African Institute of Mining and Metallurgy, Johannesburg.

Gy, P.M. 1973. The sampling of broken ores - a review of principles and practice. Proceedings of Geological, Mining and Metallurgical Sampling: 1973. Institution of Mining and Metallurgy, London. pp. 194-205, 261-263.

Gy, P.M. 1979. Sampling of particulate materials, theory and practice. Developments in Geomathematics 4. Elsevier, Amsterdam. 430 pp.
Gy, P.M. 1982. Sampling of Particulate Materials, Theory and Practice. 2nd revised edn. Elsevier, Amsterdam.

Gy, P.M. 1992. Sampling of heterogeneous and dynamic material systems. Theories of heterogeneity, Sampling and Homogenising. Elsevier, Amsterdam.

Gy, P.M. 1995. Introduction to the theory of sampling. Part 1: Heterogeneity of a population of uncorrelated units. TrAC Trends in Analytical Chemistry, vol. 14 , no. 2. pp. $67-76$.

Gy, P.M. 1998. Sampling for Analytical Purposes: The Paris School of Physics and Chemistry. Royle, A.G. (trans.). Wiley, New York. 153 pp.

Hallbauer, D.K. and Joughin, N.C. 1972. An investigation into the distribution, size and shape of gold particles in some Witwatersrand reefs and their effect on sampling procedures. Research report no. 43/72. Chamber of Mines of South Africa, Johannesburg. pp. 12-20.

HARRISON, R.N. 1952. Comment on the mine call factor. Journal of the Institute of Mine Surveyors of South Africa, Johannesburg. pp. 102-104

LERM, C. 1994. Evaluation of chip sampling vs the gold analyzer. Journal of the Institute of Mine Surveyors of South Africa. vol. XXVII, no. 5. pp. 77-87.

MAGRI, E.J. and McKENNA, P. 1986. A geostatistical study of diamond-saw sampling versus chip sampling. Journal of the South African Institute of Mining and Metallurgy, vol. 107. pp. 36-37.

MinNITT, R.C.A. 2007. Sampling: the impact on costs and decision making. Journal of the Southern African Institute of Mining and Metallurgy, vol. 107 , no. 7 . pp. 451-462.

MulLER, T.F. 1950. Underground gold losses at Rand Leases. Association of Mine Managers of the Transvaal.

Sichel, H.S. and RowLAND, R.ST.J. 1961. Recent advances in mine sampling and underground valuation practice in South African Goldfields. Transactions of the 7th Commonwealth Mining and Metallurgical Congress, Johannesburg, 10 April-7 May 1961. South African Institute of Mining and Metallurgy, Johannesburg. pp. 1-21.

STORRAR, C.D. 1987. South African Mine Valuation. Chamber of Mines of South Africa, Johannesburg. pp. 24-81.

Zuвко, A. 2003. Treasury of Spiritual Wisdom (a Collection of 10,000 Powerful Quotations for Transforming Your Life). Motilal Banarsidass, New Delhi, India. 